Technical Note

\title{
Initial experience of using an iron-containing fiducial marker for radiotherapy of prostate cancer: Advantages in the visualization of markers in Computed Tomography and Magnetic Resonance Imaging
}

\author{
Osamu TANAKA ${ }^{1, a}$, Takayoshi IIDA ${ }^{1}$, Hisao KOMEDA², Masayoshi TAMAKI ${ }^{2}$, Kensaku SEIKE ${ }^{2}$, Daiki KATO², \\ Takamasa YOKOYAMA ${ }^{1}$, Shigeki HIROSE ${ }^{1}$, Daisuke KAWAGUCH ${ }^{1}$ \\ ${ }^{1}$ Department of Radiation Oncology, Gifu Municipal Hospital, 7-1 Kashima-cho, Gifu City Gifu, 500-8513, Japan \\ ${ }^{2}$ Department of Urology, Gifu Municipal Hospital, 7-1 Kashima-cho, Gifu City Gifu, 500-8513, Japan \\ ${ }^{a}$ E-mail address: c.bluered@gmail.com
}

(received 28 August 2016; revised 3 November 2016 and 16 November 2016; accepted 22 November 2016)

\begin{abstract}
Visualization of markers is critical for imaging modalities such as computed tomography (CT) and magnetic resonance imaging (MRI). However, the size of the marker varies according to the imaging technique. While a large-sized marker is more useful for visualization in MRI, it results in artifacts on CT and causes substantial pain on administration. In contrast, a small-sized marker reduces the artifacts on CT but hampers MRI detection. Herein, we report a new ironcontaining marker and compare its utility with that of non-iron-containing markers. Five patients underwent CT/MRI fusion-based intensity-modulated radiotherapy, and the markers were placed by urologists. A Gold Anchor ${ }^{\mathrm{TM}}$ (GA; diameter, $0.28 \mathrm{~mm}$; length, $10 \mathrm{~mm}$ ) was placed using a $22 \mathrm{G}$ needle on the right side of the prostate. A VISICOIL ${ }^{\mathrm{TM}}$ (VIS; diameter, $0.35 \mathrm{~mm}$; length, $10 \mathrm{~mm}$ ) was placed using a $19 \mathrm{G}$ needle on the left side. MRI was performed using T2*-weighted imaging. Three observers evaluated and scored the visual qualities of the acquired images. The mean score of visualization was almost identical between the GA and VIS in radiography and cone-beam CT (Novalis Tx). The artifacts in planning CT were slightly larger using the GA than using the VIS. The visualization of the marker on MRI using the GA was superior to that using the VIS. In conclusion, the visualization quality of radiography, conebeam CT, and planning CT was roughly equal between the GA and VIS. However, the GA was more strongly visualized than was the VIS on MRI due to iron containing.
\end{abstract}

Key words: prostate radiotherapy; image-guided; MRI; fiducial marker.

\section{Introduction}

The precision of radiotherapy for prostate cancer has been improving, and intensity-modulated radiotherapy (IMRT) is commonly performed using fiducial markers [1-3], because the treatment is repeated and markers capable of being depicted on magnetic resonance imaging (MRI) are necessary. However, there are many fiducial markers in the world and they can sometimes be difficult to recognize on MR. If bigger marker is well depict on MRI, but the artifact on CT is not sometimes tolerable. Beside bigger marker needs needle of big in diameter and it causes pain and seeding of tumor. Before we began the present study, the $0.35 \mathrm{~mm} \times 10 \mathrm{~mm}$ and $0.5 \mathrm{~mm} \times 10 \mathrm{~mm}$ VIS markers had been well recognized visually on cone-beam CT in all cases. Herein, we report our findings regarding the utility of a $0.5 \%$-iron-containing fiducial marker (Gold Anchor ${ }^{\mathrm{TM}}$ [GA]; Naslund Medical AB, Huddinge, Sweden) versus a commonly used linear fiducial marker (VISICOIL ${ }^{\mathrm{TM}}$ [VIS]; RadioMed Corporation, Bartlett, TN, USA) in five patients at our hospital.

\section{Materials and Methods}

From June to July 2016, five patients participated in this study. All of the patients provided written informed consent. The fiducial marker was placed by urologists via the transperineal method under local anesthesia three weeks before CT and MRI examinations. The VIS was placed on one side of the prostate, and the GA was placed on the opposite site.

The GA was $0.28 \mathrm{~mm}$ in diameter and $10 \mathrm{~mm}$ in length and had a winding, zigzag shape that could be bent to make the marker spherically shaped (Figure 1). The VIS was $0.35 \mathrm{~mm}$ in diameter and $10 \mathrm{~mm}$ in length and was linear, as is most common. The GAs were inserted using $22 \mathrm{G}$ needles, and the VISs were inserted using $19 \mathrm{G}$ needles (the thinnest needle available for the VIS in Japan). Patients on anticoagulants were excluded from the study. Three weeks after the insertion of the GA and VIS, plain radiograph, computed tomography (CT) and MRI were performed. 


\section{VISICOIL $0.35 \mathrm{~mm}$}
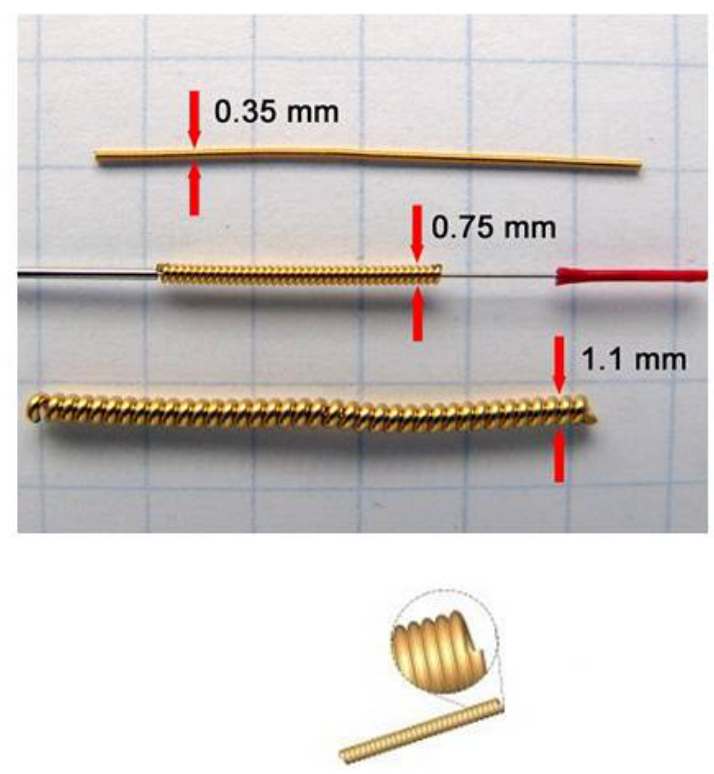

Gold Anchor $0.28 \mathrm{~mm}$

Figure 1. Marker characteristics. The Gold Anchor can be used with needles as thin as $25 \mathrm{G}$ and placed spherically. It contains $0.5 \%$ iron and is highly visible on MRI. The VISICOIL is a coiled, straight, flexible linear marker requiring a $22 \mathrm{G}$ needle, and it exhibits little migration.

The patients drank $200 \mathrm{ml}$ of water 30 minutes before undergoing CT (Optima CT580; GE Medical Systems, Milwaukee, WI, USA) and MRI (Intera 1.5 Nova; Philips Medical Systems, Eindhoven, The Netherlands) and provided urine samples. MRI was performed within 20 minutes after CT. All of the patients were given butylscopolamine to stop bowel movements.

MRI was performed with $3 \mathrm{~mm}$ section thickness, no intersection gaps, and a $16-\mathrm{cm}$ field of view using a cardiac coil. The sequence was as follows: T1-WI, T2-WI, T2*2D, $\mathrm{T} 2 * 3 \mathrm{D}$, and contrast-enhanced T1-WI. The details of the modalities are described below.

\section{Image acquisition}

- T1-weighted imaging (T1-WI): T1-weighted spin-echo. Repetition time (TR)/echo time (TE) range in milliseconds: 400-650/8; number of averages (NA): 4; number of phaseencoding steps (PESs): 192; number of frequency-encoding steps (FESs): 240; typical spatial resolutions (TPRs) of frequency/phase: 0.67/0.83.

- T2-weighted imaging (T2-WI): T2-weighted fast spin-echo. TR/TE: 4000/80; NA: 4; PESs: 205; FESs: 256; TPRs of frequency/phase: 0.63/0.80.

- T2* two-dimensional-weighted imaging (T2*-2D): T2*weighted gradient echo. TR/TE: 700/18; NA: 2; PESs: 205; FESs: 256; TPRs of frequency/phase: 0.63/0.78.

- $\mathrm{T} 2 *$ three-dimensional-weighted imaging (T2*3D): T2*-3Dweighted gradient echo. TR/TE1/DTE: 37/14/7.3; NA: 2; PESs: 218; FESs: 272; TPRs of frequency/phase: 0.55/0.54.
- Contrast-enhanced T1-weighted imaging (contrast-enhanced T1-WI): Contrast enhanced T1-weighted spin-echo. TR/TE: 400-650/8; NA: 4; PESs: 192; FESs: 240; TPRs of frequency/phase: $0.67 / 0.83$.

- Planning CT: thickness $1.25 \mathrm{~mm}$, pixel 512 x 512, $120 \mathrm{kV}$ and $250 \mathrm{~mA}$, Field of View $50 \times 50 \mathrm{~cm}$.

- Cone-beam CT: thickness $2.5 \mathrm{~mm}$, pixel 384 x 384, 125kV and $80 \mathrm{~mA}$, Field of View $45 \mathrm{x} 45 \mathrm{~cm}$.

We selected the images of T2-WI, T2*2D, and T2*3D among the five MRI sequences because these showed the best visualization. We examined the degree of artifacts on CT and marker visualization on MRI.

The radiotherapy instrument used was a Novalis Tx system (Varian Medical Systems, Inc., Palo Alto, CA, USA).

\section{Evaluation of images}

The degree of recognition of the prostatic outline despite artifacts on CT was scored as follows: 1, poor; 2, slightly poor; 3 , neutral; 4, marginally good; and 5, excellent. The degree of recognition of the marker itself on the prostate on MRI was scored as follows: 1, poor; 2 , slightly poor; 3 , neutral; 4, marginally good; and 5, excellent. The degree of recognition of the marker and the prostatic outline on MRI was analyzed, and we adopted the best sequences among T2-WI, T2*2D-WI, and T2*3D-WI. Urologists also evaluated the visibility of the marker and needle on transrectal echography. The Institutional Review Board approved this study (No. 265), and the trial was registered on the UMIN Clinical Trials Registry (Clinical Trial Registration No. 21510). 


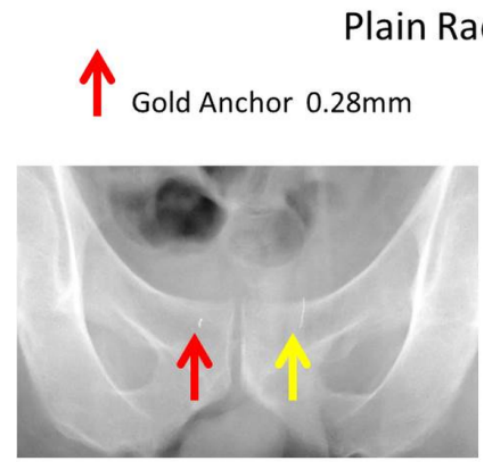

Patient 1

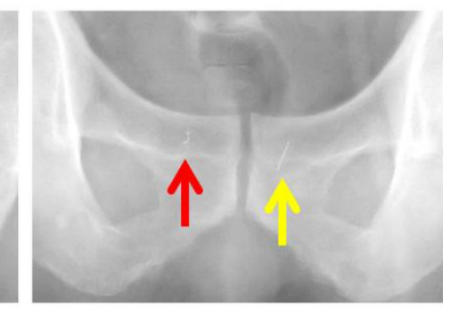

Patient 2

Figure 2. Visibility on plain radiography: Gold Anchor: $0.28 \mathrm{~mm}$; VISICOIL: $0.35 \mathrm{~mm}$. The recognition of both markers was equally good.

\section{Patient 1}

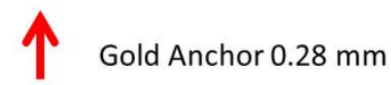

VISICOIL $0.35 \mathrm{~mm}$

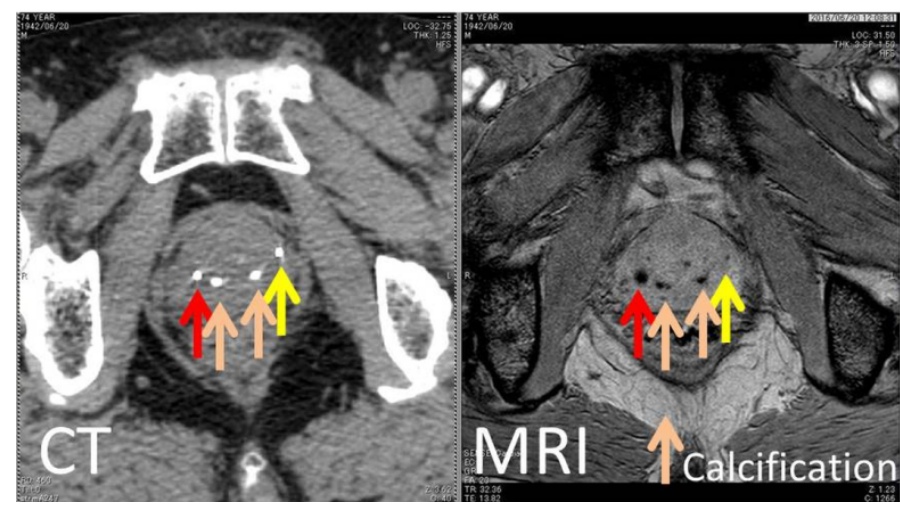

Figure 3. A comparison of the MRI visibility of the Gold Anchor, VISICOIL, and calcification. Both markers showed similar degrees of artifacts on CT. The Gold Anchor and calcification were more visible than VISICOIL. Both the Gold Anchor and calcification were well-depicted.

\section{Patient 2}

\section{A Gold Anchor $0.28 \mathrm{~mm}$}

VISICOIL $0.35 \mathrm{~mm}$

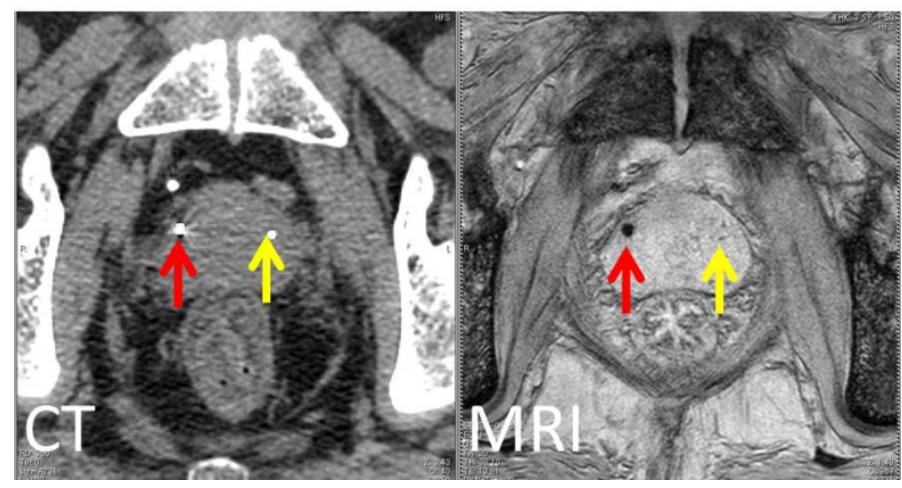

Figure 4. VISICOIL: A yellow arrow. The marker was not recognized on MRI.

\section{Patient 3 \\ $\uparrow$ Gold Anchor $0.28 \mathrm{~mm}$ \\ VISICOIL $0.35 \mathrm{~mm}$}

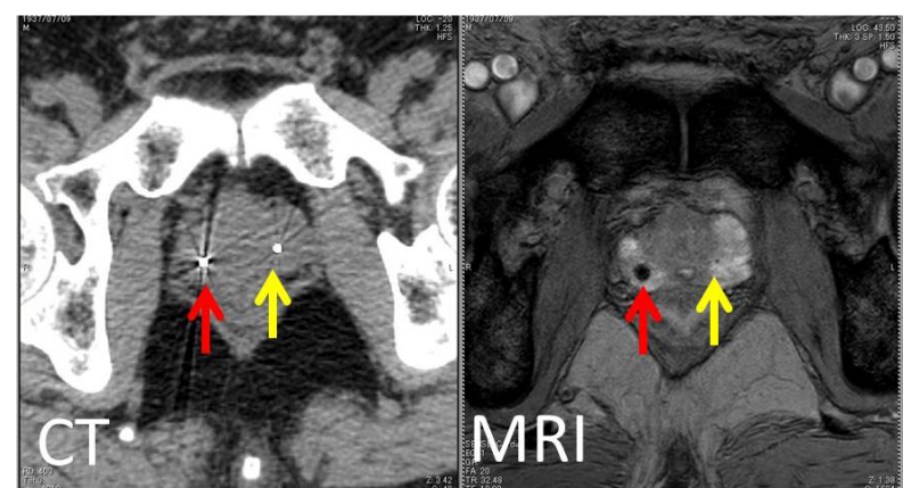

Figure 5. Gold Anchor: A red arrow. The artifacts on CT was bigger than VISICOIL.

\section{Results}

We did not precisely conduct any statistical analyses in this study because of limited number of patients. We obtained pelvis plain radiography using either the GA or VIS (Figure 2). On CT, the GA produced moderately bigger artifacts than did the VIS, but the GA did not influence the visualization of the prostate or surrounding organs (Figures 35). However, the visibility external contour of prostate on MRI was clearly better when using the GA than when using the VIS (Figure 4). The GA had a visibility similar to that of coarse calcification (Figure 3). In contrast, the VIS $(0.35 \mathrm{~mm})$ was slightly difficult to visualize on MRI.

The mean score of artifacts on CT was 3.4 with VIS and 2.5 with GA. The mean score of depiction as signal void on MRI was 2.2 with VIS and 4.4 with GA. The outlines of the prostate showed little difference between the two markers.

Both markers could be recognized equally well on transrectal echography by urologist.

\section{Discussion}

The clinical results of radiotherapy depend on the reproducibility of high-precision techniques such as IMRT throughout the radiotherapy course, because we monitor the dynamics and increase the dosage to the prostate or reduce the dosage to the surrounding normal tissues based on these findings. In addition, real-time tracking can reduce the risk of complications associated with IMRT at the location of the prostate, which varies within the body.

However, the prostatic outlines are indistinct, and treatment adjustment and contouring of the organs can prove difficult when using CT alone. As such, MRI is often used to compensate for any shortcomings of CT [4,5]. Because the prostatic outlines are clearer on MRI, it is performed after CT and is registered under the guidance of markers.

Marker sizes vary globally and range in diameter from $0.35 \mathrm{~mm}$ to $1.1 \mathrm{~mm}$ and in length from $10 \mathrm{~mm}$ to $30 \mathrm{~mm}$. At 
present, a diameter of $0.75 \mathrm{~mm}$ and length of $0.5 \mathrm{~mm}$ are most frequently used in Japan. The recognition precision on MRI increases with marker size, thereby simplifying the treatment. However, the recognition precision on CT decreases with increasing marker size, as artifacts begin to appear when a large volume of metal is present. In addition, the prostate is a small organ, and the presence of metal either in the marker or within the organ itself may influence the dose distribution. Tanaka et al. developed an optimal MRI sequence based on marker size, and a marker diameter of $0.35 \mathrm{~mm}$ has since been adopted at our hospital $[4,5]$.

In February 2016, however, a marker with a diameter of $0.28 \mathrm{~mm}$, a $22 \mathrm{G}$ needle, and iron-containing markers became available in Japan. The GA used in the present study contains $0.5 \%$ iron, and its visibility on MRI is reported to be superior to that of non-iron-containing markers. Iron-containing markers have been widely used in other countries since 2010, and previous studies have reported fewer artifacts on CT and increased visibility on MRI when using these markers than when using the conventional gold markers.

Most facilities use 0.35 - to 0.75 -mm-diameter markers, but recently, by virtue of repeated experience, the $0.5-\mathrm{mm}$ diameter marker has been preferred. We employed a $0.35-\mathrm{mm}$ marker because it was well recognized on cone-beam CT and helped reduce the artifacts on CT. To our knowledge, no previous studies have compared the outcomes of the GA with those of other markers in the same individual, albeit we did find some reports of phantom studies [5].

At present only $19 \mathrm{G}$ needles can be sold in Japan, and 22G needles for VIS are still not available. Nevertheless, in this initial experience of five cases, the chalybeate marker greatly contributed to registration using MRI for radiation treatment planning in clinical practice.

\section{Conclusion}

An iron-containing marker was extremely useful in image registration (planning $\mathrm{CT}$ to $\mathrm{MRI}$ and planning $\mathrm{CT}$ to conebeam CT). Bleeding and pain can be avoided by using a thin needle, and the marker was able to be recognized on prostatic MRI even when using a thin $22 \mathrm{G}$ needle. The artifacts on CT were tolerable for both GA and VIS. The present findings suggest that the Gold Anchor will indeed be useful in daily practice.

\section{References}

[1] Chan MF, Cohen GN, Deasy JO. Qualitative evaluation of fiducial markers for radiotherapy imaging. Technol Cancer Res Treat. 2015;14(3):298-304.

[2] $\mathrm{Ng} \mathrm{M}$, Brown E, Williams A, et al. Fiducial markers and spacers in prostate radiotherapy: current applications. BJU Int. 2014;113(S2):13-20.

[3] Gurney-Champion OJ, Lens E, van der Horst A, et al. Visibility and artifacts of gold fiducial markers used for image guided radiation therapy of pancreatic cancer on MRI. Med Phys. 2015;42(5):2638-2647.

[4] Tanaka O, Hayashi S, Matsuo M, et al. Comparison of MRI-based and CT/MRI fusion-based postimplant dosimetric analysis of prostate brachytherapy. Int J Radiat Oncol Biol Phys. 2006;66(2):597-602.

[5] Tanaka O, Hattori M, Hirose S. Comparison of the MRI sequences in ideal fiducial marker-based radiotherapy for prostate cancer. Radiother Oncol. 2016;119(Supplement1):S858-S859. 